\title{
INTERPRETACIÓN DE LAS REACCIONES DE OXIDACIÓN-REDUCCIÓN POR LOS ESTUDIANTES. PRIMEROS RESULTADOS
}

\author{
BUESO, A. ${ }^{1}$, FURIÓ, C. $^{2}$ y MANS, C. $^{3}$ \\ (1) I.B. Vilumara, L'Hospitalet. \\ (2) Servei de Formació del Professorat de la Universitat de València. \\ (3) Departament d'Enginyeria Química i Metal-lúrgia. Universitat de Barcelona.
}

\begin{abstract}
SUMMARY
This paper shows a survey on the interpretations given by students to the oxidation -reduction reactions. The research tried to check whether by the end of secondary schools studies the subjects had reached the understanding of such reactions as processes where an electronic interchange in the reacting chemical takes place.
\end{abstract}

\section{INTRODUCCIÓN}

La línea de investigación sobre los esquemas conceptuales en el dominio de la enseñanza de la Química no parece tan desarrollada como en el caso de la Física o de la Biologia; no obstante recientemente están aflo. rando en la literatura algunos trabajos en el contexto de las reacciones químicas (Carbonell y Furió, 1987) o en el de la conservación de la masa en estos procesos (Driver, 1985). Hay que reconocer que los conocimientos previos de los alumnos en el comportamiento químico de la materia no deben suponer barreras episte. mológicas importantes a su aprendizaje y, por tanto, adquieren un mayor relieve las estrategias de enseñanza utilizadas por el profesorado (Hemández y Furió, 1987). Dentro de éstas será importante averiguar la eficacia de nuestros métodos y contrastar cuánto aprendizaje significativo se ha logrado y en qué nivel de conocimientos se encuentran los estudiantes cuando con* cluyen las enseñanzas medias.

En este trabajo se ha realizado un sondeo de las interpretaciones que los alumnos dan a las reacciones de oxidación-reducción. Este estudio se ha dirigido, en particular, a comprobar si los estudiantes al concluir sus estudios secundarios habian o no alcanzado una comprensión de estas reacciones como procesos en los que hay un intercambio electrónico en las especies químicas reaccionantes.

\section{EMISION DE HIPÓTESIS Y SU FUNDA- MENTACIÓN TEÓRICA}

La historia de la Química en cuanto a estas concepciones ha demostrado la existencia progresiva de varias etapas en las cuales estas teorias se han ido haciendo más inclusivas. Así vemos que, a excepción de la época precientífica donde fue ganando cuerpo la idea sustancial y material de los gases, así como su diferencia. ción, en una primera etapa se interpretaron las reacciones de oxidación de los metales, la reducción de sus óxidos y las combustiones orgánicas como reacciones típicas de oxidación-reducción, cuya caracteristica esencial era la intervención del oxígeno del aire en la oxidación y su eliminación en la reducción (Kuhn, 1971).

Más adelante y al descubrirse efectos descriptivos si. milares de otros gases como los halógenos (tanto o más reactivos que el oxigeno) sobre los metales y demás sustancias y, sobre todo, al superarse la idea del átomo daltoniano indivisible y admitirse la existencia de electrones en su interior, es cuando va abriéndose paso la nueva concepción de los procesos redox. No obstante, habrá que esperar a la emergencia de las teorías del enlace químico, a principios de siglo, para que se afiance la interpretación de las reacciones de oxidaciónreducción como intercambio electrónico.

Pues bien, si aceptamos el marco general piagetiano que sugiere el isomorfismo entre el crecimiento de los conocimientos científicos y el desarrollo de las funciones cognitivas del estudiante (Piaget, 1975), en este contexto no es difícil presuponer que el nivel adquirido por los alumnos de Química que terminan sus estudios me. dios o que inician los universitarios en Ciencias se encuentre en el primer estadio de aquellas concepciones; es decir, que tengan, a lo sumo, unas representaciones mentales respecto de las reacciones redox como proce. sos de intercambio de oxígeno. Ello significa que no 
interpretarán estos procesos como de transferencia electrónica, a pesar de haberlos estudiado en sus cursos de Química.

El fundamento de estas hipótesis habrá que buscarlo en el resultado de sus experiencias sensibles y lingüísticas. Dado que en este contexto sus esquemas conceptuales habrán sido adquiridos, en su mayor parte, a través de una transmisión linguistica en el ámbito escolar, es de esperar que las variables que más hayan influido sean los contenidos de la enseñanza recibida y las metodologias utilizadas en su proceso de aprendizaje (Driver y Erickson, 1983; Gil, 1983). Si, como estimamos, los contenidos enseñados han sido reducidos y las metodologías utilizadas se basan en la transmisión verbal de los conocimientos ya elaborados, es previsible que se cumpla la siguiente hipótesis:

Las interpretaciones sobre las reacciones de oxidaciónreducción de los estudiantes que acaban sus esiudios medios estarán mucho más próximas a una concepción superficial de estas reacciones, como procesos de intercambio de oxigeno, que a la de transferencia electrónica.

Las consecuencias de esta hipótesis se manifestarán, según nuestra opinión, tanto a nivel fenomenológico cuando se les proponga la descripción de ejemplos que tengan que ver con estos procesos, como en un nivel más profundo de análisis en situaciones donde hayan de interpretar conceptualmente alguna de las reacciones en las que no intervenga el oxigeno.

\section{DISEÑO EXPERIMENTAL}

En un primer sondeo se han tomado cuatro grupos de alumnos de $3^{\circ}$ de BUP y de COU de I.B. Francesc Macià de Cornellà, y de $1^{\circ}$ de Biología y de $3^{\circ}$ de Química de la Universidad de Barcelona, y se les encuestará con preguntas abiertas y de opción múltiple con los siguientes objetivos:

a) En el nivel fenomenológico se han planteado varias preguntas. Una abierta, de asociación de ejemplos con cada uno de los conceptos de oxidación y de reducción, con el fin de analizar las categorías de respuestas y sus porcentajes respectivos según los dos niveles indicados en la hipótesis (proceso redox como intercambio de oxigeno o como transferencia electrónica). Otras dos cuestiones de opción múltiple, para ver en qué medida atribuyen masa y volumen al oxígeno del aire, en un proceso familiar como la oxidación del hierro, y asi observar el nivel de profundización adquirido en estas representaciones mentales.

b) En el aspecto conceptual se han planteado sendas cuestiones abiertas, una de ellas para que expliciten su definición general de estos procesos y otra de interpretación icónica y argumental de un proceso no familiar como la reacción de síntesis del cloruro de cinc. El análisis del contenido de las respuestas a esta úttima cues- tión debe concluir - -5i la hipótesis es vấlida- que, como máximo, abundarán interpretaciones próximas al modelo daltoniano de reacción química como distribución nueva de los átomos y, en cambio, habrá ausencia de interpretaciones como formación de iones (lo que supone intercambio electrónico) constituidos o no en una red iónica.

\section{RESULTADOS Y CONCLUSIONES}

En las Tablas I y II se muestran los porcentajes de los resultados correspondientes a las preguntas 1 y 2 , donde se pedía a los alumnos que escribiesen tres ejemplos de oxidaciones y otros tres de reducciones, respectivamen. te.

Puede observarse el gran porcentaje de ejemplos en que interviene el oxígeno, tanto para la oxiđación como para la reducción, así como el hecho que el mayor núme. ro de ejemplos corresponda a oxidaciones de metales u objetos metálicos, lo cual nos indica que la asimilación del concepto de oxidación-reducción por parte de los alumnos se encuentra en el primer estadio de desa* rrollo, es decir, la oxidación-reducción como intercambio de oxígeno y no como intercambio de electrones.

En consecuencia, dicha evolución conceptual puede observarse perfectamente entre los alumnos de BUP y COU: $51 \%$ de ejemplos referidos a oxidaciones de metales; $5,5 \%$ son ejemplos de reacciones entre un compuesto y el oxígeno, mientras que tan sólo el $2,8 \%$ de los ejemplos se refieren a oxidaciones mediante no metales, y otro $2,8 \%$ de los ejemplos mencionan la reacción de oxidación-reducción como reacción de intercambio electrónico.

Otra constatación que también podia esperarse a prio$r i$, es el mayor grado de dificultad que supone encontrar ejemplos de seducción en comparación con los ejemplos de oxidación (véanse los porcentajes de alum. nos que no consiguieron encontrar ni un solo ejemplo).

En la Tabla III se encuentran los porcentajes de las respuestas a la pregunta 3 , de opción múltiple, basada en un ejemplo familiar para los alumnos como es la oxidación del hierro al aire libre, en la que se debía comparar la masa del hierro puro inicial con la del óxido formado.

Es curioso observar cómo, a pesar de que casi la ma" yor parte de los alumnos de BUP, COU y $1^{\circ}$ de Facultad han propuesto en la pregunta anterior ejemplos de reacciones con el oxígeno, tan sólo la mitad admite que la reacción del oxigeno con el hierro hace aumentar la masa del óxido. A partir de las explicaciones dadas por los alumnos que han respondido que ambas masas eran idénticas, se puede deducir que han efectuado una in. correcta aplicación del principio de conservación de la masa, al tratarse de un sistema abierto. Por su parte, la respuesta de que el hierro pesa más que el óxido formado en la reacción puede interpretarse como una con- 
Tabla I

Porcentaje de ejemplos de oxidación propuestos por los alumnos.

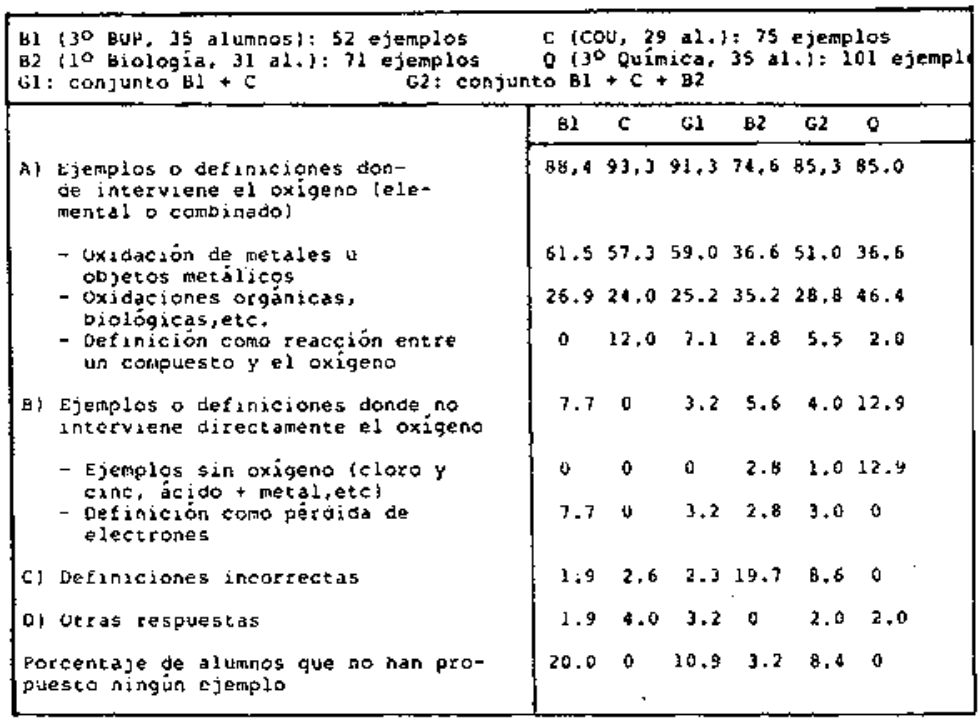

Tabla II

Porcentaje de ejemplos de reducción propuestos por los alumnos.

\begin{tabular}{|c|c|c|c|c|c|c|c|}
\hline $\begin{array}{l}81 \\
\text { B2 }\end{array}$ & $\begin{array}{l}: 34 \text { ejemplos } \\
: 44 \text { ejemplos }\end{array}$ & $\begin{array}{l}\text { ejem } \\
\text { ejem }\end{array}$ & & & & & \\
\hline \multirow{5}{*}{ A) } & & يl & $c$ & Gl & 82 & G2 & $\underline{q}$ \\
\hline & $\begin{array}{l}\text { Ejemplos o definiciones como pér- } \\
\text { dida de oxigeno }\end{array}$ & 68.4 & 49.9 & 57.4 & 34.1 & 50.0 & 39.0 \\
\hline & $\begin{array}{l}\text { - Obtención del metal a partix } \\
\text { del óxido }\end{array}$ & 36.8 & 17.8 & 25.5 & 9.1 & 20.3 & 19.5 \\
\hline & - Reacción donde se extrde oxígeno & 0 & 23.2 & 13,8 & 6.8 & 11.6 & 1,2 \\
\hline & $\begin{array}{l}\text { - Reducciones orgánicas, biolö- } \\
\text { gicas,etc. }\end{array}$ & 31.6 & 8.9 & 18.1 & 18.2 & 18.1 & 18.2 \\
\hline \multirow[t]{3}{*}{ B) } & $\begin{array}{l}\text { Ejemplos o definiciones sin inter- } \\
\text { vención directa del oxigeno }\end{array}$ & 23.6 & 3.6 & 11.7 & 18.2 & 13.8 & 56.1 \\
\hline & $\begin{array}{l}\text { - Ejemplos sin oxígeno } \\
\text { - Definición como gananca de } \\
\text { electrones }\end{array}$ & 2.6 & $\stackrel{0}{0}$ & $\begin{array}{l}0 \\
1.1\end{array}$ & 4.5 & 2.2 & $\begin{array}{r}54.9 \\
1.2\end{array}$ \\
\hline & $\begin{array}{l}\text { - Definición como assminueión de } \\
\text { la valencia }\end{array}$ & 21.0 & 3.6 & 10.6 & 13.6 & 11.6 & b \\
\hline \multirow[t]{2}{*}{ c) } & Definiciones incorrectas & 3.9 & 25.0 & 18.1 & 36.4 & 23.9 & 0 \\
\hline & $\begin{array}{l}\text { - Oefinición como pérdida de ma- } \\
\text { teria }\end{array}$ & 2.6 & 12.5 & d. 5 & 0 & 5.8 & 0 \\
\hline D) & Otras respuestas & 0 & 21.4 & 12.8 & 11.0 & 12.3 & 4.8 \\
\hline $\begin{array}{l}\text { Por } \\
\text { pro }\end{array}$ & $\begin{array}{l}\text { rcentaje de alumnos que no han } \\
\text { opuesto ningün ejemplo }\end{array}$ & 28.6 & 0 & 35.6 & 29.0 & 20.0 & 0 \\
\hline
\end{tabular}

Tabla III

«En la oxidación del hierro al aire tibre..."

Respuestas en porcentaje de alumnos.

\begin{tabular}{|c|c|c|c|c|c|c|}
\hline & B1 & $c$ & G1 & B2 & G2 & 0 \\
\hline $\begin{array}{l}\text { - El hierro pesa lo mismo que el óxido } \\
\text { de hierro obtenido } \\
\text { - El hiexro pesa más que el óxido de } \\
\text { hierro obtenido } \\
\text { - El hierro pesa menos que el óxido de } \\
\text { hierro obtenido } \\
\text { - No contestan }\end{array}$ & $\begin{array}{l}11.4 \\
40.0 \\
18.6 \\
0\end{array}$ & $\begin{array}{l}20.7 \\
13.8 \\
65.5 \\
0 .\end{array}$ & $\begin{array}{l}15.6 \\
28.1 \\
56.2 \\
0\end{array}$ & $\begin{array}{r}12.9 \\
48.4 \\
35.5 \\
3.2\end{array}$ & $\begin{array}{r}14.7 \\
34.7 \\
49.5 \\
1.1\end{array}$ & $\begin{array}{l}2.8 \\
2.8 \\
94.3 \\
0\end{array}$ \\
\hline
\end{tabular}


fusión entre los términos peso y densidad, o bien por el hecho de comparar sólo la masa de la capa superficial de óxido de hierro con la masa del resto de hierro no oxidado; mediante posteriores entrevistas, intentaremos dilucidar entre ambas suposiciones.

Comparando estos resultados con los obtenidos por Driver (1985) con alumnos de 15 años, y por Furió y Hernández (1987), resultan ser bastante coincidentes con los de la muestra de alumnos de BUP, COU y $1^{\circ}$ de Facultad. Por ejemplo, los resultados obtenidos por Driver, y citados en idéntico orden, son: $5,0 \% ; 41,0 \%$; $48,0 \%$ y $6,0 \%$.

La pregunta 4 se refería también a la oxidación del hierro, pero esta vez en el sistema cerrado de la figura 1.

figura 1

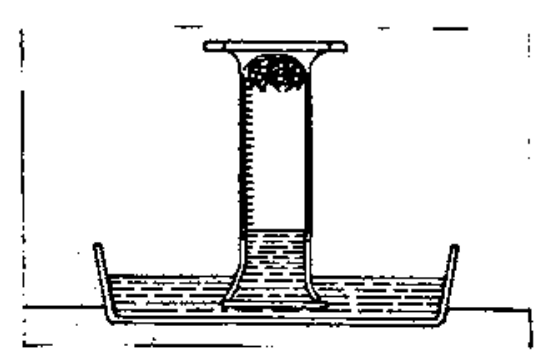

Los resultados (Tabla IV) son muy similares a los de la pregunta anterior, ya que aproximadamente un $50 \%$ de los alumnos responde que el hierro reacciona con el oxigeno del aire. Es significativo, no obstante, observar el elevado porcentaje de alumnos de $3^{\circ}$ de Química que aún se dejan influir por el oxigeno del agua.

Estos resultados sirven para poner de manifiesto la superficialidad de los conceptos adquiridos por los alumnos, ya que tan sólo la mitad de los alumnos que terminan sus estudios secundarios han comprendido un concepto que, por otra parte, les resulta tan familiar, al haberlo puesto la mayoría como ejemplo en la pregunta 1.
La pregunta 5 pedia la definición del concepto de oxidación-reducción. En la Tabla $V$ se reflejan los resultados obtenidos. Es muy indicativo que sólo el $3 \%$ de los alumnos de BUP y de COU han respondido al concepto de oxidación $\rightarrow$ reducción como reacción de transferencia electrónica, lo que confirma que este concepto no es impartido en Bachillerato, quizá porque no se imparte. En COU figura al final del programa, pero dado el condicionante de las pruebas de Selectividad, probablemente se enfoca más en su aspecto «mecánico " de aprender a igualar reacciones que en su aspecto conceptual. Corrobora dicha hipótesis el hecho que el $10 \%$ de los alumnos de este nivel definan la oxidación-reducción como reacción de transferencia de protones o de formación de iones (lo que ocurre al igualar estas reacciones por el usual método del iónelectrón). Al igual que en la pregunta anterior, es conveniente entrevistar a estos alumnos y averiguar el mo* tivo real de estas respuestas. En el caso de los alumnos de $1^{\circ}$ de Biología, si bien se observa que la mitad de estos alumnos conocen las reacciones de oxidaciónreducción como reacciones de transferencia electrónica, conviene tener en cuenta el alto porcentaje $(32,2 \%)$ que aplican erróneamente dicho concepto, al no saber si en la oxidación tiene lugar pérdida o ganancia de electrones; en definitiva, tan sólo la tercera parte de los alumnos que reconocen que se trata de una reacción de transferencia de electrones sabe aplicar correctamente el concepto, lo que lleva a incidir una vez más en la superficialidad de la asimilación de los conocimientos, no sólo en los Institutos de Bachillerato sino también en los primeros cursos de Universidad. Este hecho llega aún a ser más grave, si comparamos el $32 \%$ de respuestas incorrectas de los alumnos de $1^{\circ}$ de Bio. logía con el $4,7 \%$ de los alumnos de BUP y COU. En definitiva, se confirma la hipótesis de que los contenidos se reducen y las metodologías basadas en la transmision verbal de los conocimientos conducen a una mala asimilación del concepto actualmente aceptado de oxidación-reducción por parte de los alumnos.

Finalmente la pregunta 6 (Tabla VI) era una pregunta abierta consistente en dibujar e interpretar la reacción

Tabla IV

«En ia oxidación del hierro en el sistema cerrado de la figura..." Respuestas en porcentaje de alumnos.

\begin{tabular}{|c|c|c|c|c|c|c|}
\hline & BI & c & GI & B2 & 62 & 8 \\
\hline $\begin{array}{l}\text { a) No pasa nada } \\
\text { b) El hierro reacciona con el oxígeno } \\
\text { del agua y paja el nivel del agua }\end{array}$ & $\begin{array}{l}2.8 \\
5.7\end{array}$ & $\begin{array}{r}6.9 \\
20.7\end{array}$ & $\begin{array}{r}4.7 \\
12.5\end{array}$ & $\begin{array}{c}0 \\
22.6\end{array}$ & $\begin{array}{r}3.2 \\
15.8\end{array}$ & 11.4 \\
\hline $\begin{array}{l}\text { c) El hierro reacciona con el oxígeno } \\
\text { del aire y sube el nivel del agua }\end{array}$ & 48.6 & 37.9 & 43.7 & 51.6 & 46.3 & 74.3 \\
\hline $\begin{array}{l}\text { d) El hierro reacciona con el oxigeno } \\
\text { del aire y baja el nivel del agua }\end{array}$ & 5.7 & 10.3 & 7,8 & 3.2 & 6.3 & 8.6 \\
\hline $\begin{array}{l}\text { e) El hierro reacciona con el oxigeno } \\
\text { del agua y sube el nivel del agua }\end{array}$ & 5.7 & 6.9 & 6.2 & 9.7 & 7.4 & 0 \\
\hline $\begin{array}{l}\text { f) No lo sé } \\
\text { - No contesta }\end{array}$ & 31.4 & 17.2 & 25.0 & $\begin{array}{l}6.4 \\
6.4\end{array}$ & $\begin{array}{r}18.9 \\
2.1\end{array}$ & $\begin{array}{l}5.7 \\
0\end{array}$ \\
\hline
\end{tabular}


Tabla V

Definiciones conceptuales dadas a las reacciones Redox.

Respuestas en porcentaje de alumnos.

a) Como transfereacia electrónica loxidación como pérdida de electrones $y$ tecucción como ganancia)

B) Como intercambio de oxígeno (oxidaciön como adición de oxigeno a metales, compuestos.... y reducción como pérdicia de oxigeno\}

c) Otras respuestas aceptables (pérdida o ganancia de protones o formación de iones)

D) Definición incorrecta (oxidación como ganancia de electrones y reducción como péraida de electrones)

E) Otras respuestas no representativas (por ej.: son reacciones inversas la una cie la otra)

- kespuestas en olanco

\begin{tabular}{|cccccc|}
\hline B1 & $\mathrm{C}$ & $\mathrm{G1}$ & $\mathrm{B2}$ & $\mathrm{G2}$ & 0 \\
\hline 2.8 & 3.4 & 3.1 & 51.6 & 18.9 & 80.0 \\
51.4 & 65.6 & 57.8 & 3.2 & 40.0 & 0 \\
14.3 & 6.9 & 10.9 & 0 & 7.4 & 2.6 \\
5.7 & 3.4 & 0.7 & 32.2 & 13.7 & 0 \\
0 & 17.2 & 7.8 & 12.9 & 9.5 & 14.3 \\
25.7 & 3.4 & 15.6 & 0 & 10.5 & 2.6
\end{tabular} \mid

Tabla VI

Niveles de interpretación de la reacción entre el cloro y el cinc.

Respuestas en porcentaje de alumnos.
A) Helacionan de alguna forma (correcta o incorrectal la reacción con el concepto de oxidación - reducción como intercambio electrönico
- Interpretación casi correcta co- mo reacción redox
- Mencionan litexalmente que es un proceso redox
- Citan que hay intercambio electró- nico aunque dibujan moléculas de $\mathrm{znCl}_{2}$
- Citan que se forman enlaces iónicos - que hay tones $\mathrm{Cl}^{-} \mathrm{y}^{2 \mathrm{n}^{2}}$ ausque dibujan moléculas oe $2 n \mathrm{nl}_{2}$

B) Se concibe la reacción como redistribución regulat de átomos o moléculas de çioro en nuevos agregados de particulas de $\mathrm{ZnCl}_{2}$

- Unión simple de átomos o moléculas de cloro con atomos ajslados de cinc para formar moleculas de $2 \mathrm{nCl}_{2}$

- Redistribución de átomos o motéculas de cloro y de una red atómica de cinc con formación de moleculas de $\mathrm{anCl}$,

- Redistribución de átomos o molécu* las de cloro $y$ de una red atómica de cinc con formación de una red regular de ámos de cloro y cinc

c) Otras interpretaciones (Uniön covalente entre un átomo de cinc $y$ uno de cloro

- Respuestas incodificables - En blanco

\begin{tabular}{|c|c|c|c|c|c|}
\hline BI & c & G1 & 82 & 62 & 0 \\
\hline 21.4 & 37.9 & 23.4 & 25.0 & 24.2 & 68.6 \\
\hline 0 & 0 & 0 & 3.2 & 1.0 & 24.3 \\
\hline 0 & 0 & 0 & 6.4 & 2.1 & 37.2 \\
\hline 0 & 27.6 & 22.5 & 3.2 & 9.5 & 2.8 \\
\hline 11.4 & 10.3 & 10.9 & 12.9 & 11.6 & 14.3 \\
\hline 42.8 & 41.4 & 42.2 & 38.7 & 41.0 & 28.6 \\
\hline 28.6 & 34.5 & 31.2 & 16.2 & 26.3 & 5.7 \\
\hline 2.8 & 0 & 2.6 & 9.7 & 4.2 & 11.4 \\
\hline 11.4 & 6.9 & 9.4 & 12.9 & 10.5 & 21.4 \\
\hline 8.6 & 10.3 & 9.4 & 12.9 & 10.5 & 0 \\
\hline $\begin{array}{l}14.3 \\
22.6\end{array}$ & $\begin{array}{l}6.9 \\
3.4\end{array}$ & $\begin{array}{l}10.9 \\
14.1\end{array}$ & $\begin{array}{r}3.2 \\
19.3\end{array}$ & $\begin{array}{r}8.4 \\
15.8\end{array}$ & 2.8 \\
\hline
\end{tabular}


entre el cloro y el cinc. Al no ser ésta una reacción familiar para los alumnos, el objetivo principal consistía en ver si eran o no capaces de relacionarla con el concepto de oxidación-reducción. La tabulación de resultados se realizó tomando como respuestas positivas no sólo aquellas respuestas que mencionaban que se trataba de una reacción de oxidación-reducción, sino también todas aquellas que se referían a que tenía lugar un intercambio de electrones, formación de iones, o simplemente de un enlace iónico. Como ya se ha indicado en la formulación de la hipótesis, la categoria de respuestas asociada a una interpretación de estos procesos como intercambio de oxígeno correspondería a la redistribución daltoniana de átomos 0 moléculas.

A partir de los resultados puede observarse que prácticamente son idénticos los resultados de los alumnos de BUP y COU y los de los alumnos de $1^{\circ}$ de Facultad. Tan sólo hay un alumno entre los 95 de dicha muestra que hace una interpretación casi correcta del proceso, mientras sólo 1 de cada 9 alumnos establece alguna relación, aunque sea muy superficial, entre la reacción y el intercambio de electrones. Aquí es donde puede comprobarse la falta de aprendizaje significativo conseguido en estos alumnos que terminan sus estudios secundarios y que han seguido estrategias de aprendizaje basadas en la transmision de los conocimientos ya elaborados.

La interpretación daltoniana es efectuada por casi la mitad de los alumnos, a pesar de estar esta pregunta a continuación de otra donde se pedía explícitamente la definición del concepto de oxidación-reducción. También debe constatarse, a pesar de no ser ya el objeto fundamental de la pregunta, el bajo porcentaje de alumnos que han dibujado las redes del cinc y del cloruro de cinc.

\section{CONCLUSIONES FINALES}

A la luz de los resultados obtenidos parece plausible la hipótesis de que tanto los alumnos que acaban sus estudios medios como los alumnos de los primeros cursos de Universidad apenas han asimilado el concepto de oxidación-reducción como proceso de intercambio electrónico. En cambio, tienen asumido, tanto a nivel fenomenológico como conceptual, en forma mayoritaria, la idea de proceso redox como intercambio de oxígeno.

Actualmente se está realizando la extensión y generalización de estos resultados a muestras más amplias de alumnos de niveles inferiores ( $8^{\circ}$ de EGB y $2^{\circ}$ de BUP), que aún no han visto el concepto de oxidaciónreducción, asi como a muestras de alumnos de $3^{\circ}$ de BUP y COU de distintos Institutos de Bachillerato del Área Metropolitana de Barcelona, de diferentes ambientes socio-culturales.

En cuanto a las implicaciones didácticas del presente trabajo, pueden indicarse las siguientes:

La necesidad de hacer consciente al profesorado de la importancia y de las dificultades intrínsecas del tema, asi como de la necesidad de relacionarlo estrechamente con el tema del enlace químico, y dedicarle en la pro. gramación de la asignatura el suficiente tiempo, tanto en el aula como en el laboratorio.

La necesidad de cambiar la metodología usada tradicionalmente por el profesorado, con objeto de lograr un aprendizaje significativo y no superficial como se efectúa. Para ello habrá que tener en cuenta los conceptos previos del alumnado, que no suponen para este tema ninguna barrera epistemológica, ya que la mayor parte de ellos tiene ya asumido, si bien superficialmente, el concepto de oxidación-reducción como intercambio de oxigeno. 


\section{REFERENCIAS BIBLIOGRÁFICAS}

CARBONELL, F., y FURIO, C., 1987, Opiniones de los adolescentes respecto del cambio sustancial en las reacciones quimicas, Ensenanza de las Ciencias, 5, 1, 3-9.

DRIVER, R., 1985, Beyond appearence: the conservation of matter under physical and chemical transformations, Children's Ideas in Science. (Open University Press: Milton Keynes).

DRIVER, R. y ERICKSON, G., 1983, Theories in action: some theoretical and empirical issues in the study of the students' frameworks in science, Studies in Science Education, 10 , pp. 37-60.
GlL, D., 1983, Tres paradigmas básicos en la enseñanza de las ciencias, Enseñanza de las Ciencias, 1, 26-33.

HERNÁNDEZ, J. y FURIO, C., 1987, Instabilité des conceptions alternatives des élèves du primajre et du secondaire sur les gaz, Actes des IX Journées Internationales sur l'Education Scientifique, Chamonix (en prensa).

KUHN, T.S., 1971, Estructura de las revoluciones cientificas. (Fondo de Cultura Económica).

PIAGET, J., 1975, Introducción a la Epistemología Genética. (Paidos: Buenos Aires). 